\title{
Malarial anemia: digestive vacuole of Plasmodium falciparum mediates complement deposition on bystander cells to provoke hemophagocytosis
}

\author{
Prasad Dasari $\cdot$ Anja Fries $\cdot$ Sophia D. Heber $\cdot$ Abdulgabar Salama $\cdot$ \\ Igor-Wolfgang Blau • Klaus Lingelbach • Sebastian Chakrit Bhakdi • \\ Rachanee Udomsangpetch $\cdot$ Michael Torzewski $\cdot$ Karina Reiss $\cdot$ Sucharit Bhakdi
}

Received: 16 May 2014 / Accepted: 19 June 2014 / Published online: 2 July 2014

(C) Springer-Verlag Berlin Heidelberg 2014

\begin{abstract}
The digestive vacuole (DV) of Plasmodium falciparum, which is released into the bloodstream upon rupture of each parasitized red blood cell (RBC), was recently discovered to activate the alternative complement pathway. In the present work, we show that $\mathrm{C} 3$ - and $\mathrm{C} 5$-convertases assembling on the parasitic organelle are able to provoke deposition of activated C3 and C5b-9 on non-infected bystander erythrocytes. Direct contact of DVs with cells is mandatory for the effect, and bystander complement deposition occurs focally, possibly at the sites of contact. Complement opsonization promotes protracted erythrophagocytosis by human macrophages, an effect that is magnified
\end{abstract}

P. Dasari · A. Fries $\cdot$ S. D. Heber · S. Bhakdi $(\square)$ Department of Medical Microbiology and Hygiene, University Medical Center, Johannes Gutenberg University Mainz, Hochhaus Augustusplatz, 55202 Mainz, Germany

e-mail: sbhakdi@uni-mainz.de

\section{A. Salama · I.-W. Blau}

Department of Transfusion Medicine, Campus Virchow Clinic, Charité University Medical Clinic, Campus Benjamin Franklin, Berlin, Germany

\section{K. Lingelbach}

Department of Parasitology, Faculty of Biology,

Philipps University, Marburg, Germany

S. C. Bhakdi · R. Udomsangpetch

Department of Pathobiology, Faculty of Science,

Mahidol University, Bangkok, Thailand

\section{Torzewski}

Department of Laboratory Medicine, Robert-Bosch-Hospital,

Stuttgart, Germany

K. Reiss

Department of Dermatology, Christian Albrechts University Kiel, Kiel, Germany when ring-stage infected RBCs with reduced CD55 and CD59, or paroxysmal nocturnal hemoglobinuria (PNH)RBCs lacking these complement inhibitors are employed as targets. Bystander attack can also directly induce lysis of PNH-RBCs. Direct evidence for complement activation and bystander attack mediated by DVs was obtained through immunohistochemical analyses of brain paraffin sections from autopsies of patients who had died of cerebral malaria. C3d and the assembled C5b-9 complex could be detected in all sections, colocalizing with and often extending locally beyond massive accumulations of DVs that were identified under polarized light. This is the first demonstration that a complement-activating particle can mediate opsonization of bystander cells to promote their antibody-independent phagocytosis. The phenomenon may act in concert with other pathomechanisms to promote the development of anemia in patients with severe malaria.

Keywords P. falciparum - Malarial anemia . Complement activation - Bystander attack - Digestive vacuole $\cdot$ Hemophagocytosis

\section{Introduction}

Malarial anemia evolves through extravascular elimination of unparasitized erythrocytes by splenic macrophages, concomitant with bone marrow dysfunction and ineffective erythropoiesis [1-3]. Early studies demonstrated a shortened lifespan of platelets [4] and uninfected erythrocytes [5] in non-immune patients with falciparum malaria [6]. Ingestion of non-infected and ring-stage infected RBCs by splenic macrophages has been documented by electron microscopy [7], and evidence for hemophagocytosis by bone marrow macrophages is available $[8,9]$. Interaction 
of CD36 with surface-exposed parasite proteins promotes macrophage uptake of infected cells [10]. Moreover, the latter harbor oxidized membrane lipids promoting erythrophagocytosis that can transfer to non-infected cells to increase their macrophage uptake [11]. The contribution of immunological mechanisms to erythrophagocytosis remains less clear. $\mathrm{C} 3 \mathrm{~d}$ is detectable on a fraction of circulating non-infected erythrocytes by the direct Coombs test and flow cytometry $[12,13]$. However, since these cells do not carry IgG, the origin and significance of bound $\mathrm{C} 3$ have remained obscure $[12,13]$.

The parasite digestive vacuole (DV) was previously discovered to activate the alternative complement pathway [14]. We now show that DVs mediate complement deposition on bystander cells to promote protracted, antibodyindependent hemophagocytosis. In synergy with other pathomechanisms, the phenomenon may contribute to development of anemia in patients with severe malaria.

\section{Materials and methods}

RBCs were acquired from citrated blood of healthy donors and from 4 patients with PNH from whom written informed consent had been obtained in accordance with the Declaration of Helsinki. The percentage of PNH cells was 50-60\% in two patients who were on treatment with Eculizumab, and 30-40\% in two patients who were not on treatment. Plasma was obtained by centrifugation at $800 \times g$ for $10 \mathrm{~min}$ at $4{ }^{\circ} \mathrm{C}$, and cells were washed four times with phosphate-buffered saline (PBS) and used within $24 \mathrm{~h}$. Heat-inactivation was performed by incubating plasma at $56^{\circ} \mathrm{C}$ for $30 \mathrm{~min}$.

\section{Monocyte-derived macrophages}

Human peripheral blood mononuclear cells were isolated from buffy coats and macrophage cultures prepared essentially as described [15]. Monocytes were resuspended at $1 \times 10^{6} / \mathrm{ml}$ in RPMI 1640 supplemented with $100 \mathrm{U} / \mathrm{ml}$ penicillin and $100 \mu \mathrm{g} / \mathrm{ml}$ streptomycin (PenStrep, Gibco). One million cells were seeded in each well on glass cover slips in 24-well tissue culture plates and allowed to adhere for $1 \mathrm{~h}$. Non-adherent cells were removed, and cells were then grown for 5-6 days in RPMI 1640 medium supplemented with $10 \%$ fetal calf serum (Biochrom AG, Berlin, Germany) and $50 \mathrm{ng} / \mathrm{ml} \mathrm{rhM}-\mathrm{CSF}$ (R\&D Systems, Minneapolis, USA).

Parasite culture

Plasmodium falciparum 3D7 was cultured in human A+ erythrocytes at $5 \%$ hematocrit in a standard gas mixture of $5 \% \mathrm{O}_{2}, 5 \% \mathrm{CO}_{2}$, and $90 \% \mathrm{~N}_{2}$ in RMPI 1640 (Invitrogen, Karlsruhe, Germany) medium at $37{ }^{\circ} \mathrm{C}$ [16]. The medium was supplemented with $10 \%$ heat-inactivated $\mathrm{A}+$ serum, $25 \mathrm{mM}$ sodium bicarbonate (Sigma-Aldrich, Hamburg, Germany), $200 \mu \mathrm{M}$ hypoxanthin (C.C. PRO, Oberdorla, Germany) and $25 \mu \mathrm{M}$ gentamicin (Carl Roth GmbH, Karlsruhe, Germany). Parasite culture was tightly synchronized by two successive treatments with $5 \%$ sorbitol at $4 \mathrm{~h}$ intervals [17].

DVs were isolated, enumerated and banked in $50 \%$ glycerol at $-20{ }^{\circ} \mathrm{C}$ as described $[14,18]$. Immediately prior to use, they were washed twice in veronal-buffered saline (VBS) (Virion/Serion, Würzburg, Germany) and, unless otherwise stated, employed at a 1:1 ratio with target cells.

\section{Enrichment of ring-stage parasites}

Ring-stage parasites were separated from non-infected RBCs in a Percoll-sorbitol discontinuous gradient [19]. Briefly, tightly synchronized cultures containing 8-10\% ring-stage parasitized $\mathrm{RBCs}$ were centrifuged at $800 \times g$ for $5 \mathrm{~min}$. Five hundred microliters of packed erythrocytes were resuspended in $2 \mathrm{ml}$ PBS-sorbitol and layered on top of a gradient containing $4 \mathrm{ml} 90 \%, 3 \mathrm{ml} 85 \%$, $3 \mathrm{ml} 80 \%$ and $1 \mathrm{ml} 70 \%$ Percoll-sorbitol. The gradient was centrifuged at $10,000 \mathrm{rpm}$ for $20 \mathrm{~min}$ using a fixed angle SS34 rotor in a Sorvall RC-2B centrifuge. Ringstage infected erythrocytes were retrieved and washed twice with VBS.

Complement deposition on bystander cells

$1 \times 10^{8}$ DVs were washed twice with VBS in Eppendorf tubes. To obtain a ratio of DV:RBC of approximately $1: 1$, $20 \mu \mathrm{l}$ of $50 \%$ RBCs was added to the pellet and the reaction started by the addition of $200 \mu \mathrm{l}$ autologous active or heat-inactivated plasma, in which DVs and cells were resuspended and incubated at $37{ }^{\circ} \mathrm{C}$ for $1 \mathrm{~h}$ in an Eppendorf 5436 thermomixer, rotated at $1,000 \mathrm{rpm} / \mathrm{min}$. As a further control, RBCs were incubated in plasma without DVs. In all cases, results obtained in these controls matched those obtained with heat-inactivated plasma plus DVs, and so results of only one control are shown in each experiment. When PNH-RBCs were employed for subsequent erythrophagocytosis experiments, the concentration of plasma was reduced to $50 \%$ in order to minimize hemolysis during incubation with the DVs. After coincubation, RBCs and DVs were either centrifuged at $17,000 \times g$ for $30 \mathrm{~s}$ for determination of hemolysis, or, for subsequent erythrophagocytosis experiments, the bulk of DVs was removed by washing four times at $400 \times g$ for $40 \mathrm{~s}$ in a table-top centrifuge. Samples destined for Western blot analyses were prepared as detailed below. 
Filter experiments

Two-compartment culture plates $\left(6.5-\mathrm{mm}\right.$ Transwell ${ }^{\circledR}$ with 3.0- $\mu \mathrm{m}$ pore polycarbonate membrane insert, Corning Incorporated, Corning, USA) were employed wherein the filters separated RBCs in the lower compartment from the DVs in the upper compartment, with active plasma present in both. DVs were omitted in the controls.

\section{Immunofluorescent staining for $\mathrm{C} 3 \mathrm{~d}$}

Washed RBCs were blocked with $3 \%$ BSA in PBS for $30 \mathrm{~min}$ at RT. After washing, RBCs were incubated with polyclonal rabbit anti-human $\mathrm{C} 3 \mathrm{~d}$ antibody (DakoCytomation Gmbh, Hamburg, Germany) in blocking buffer at 1:50 dilution for $1 \mathrm{~h}$ at RT, followed by incubation with Alexa Fluor 594-conjugated goat anti-rabbit IgG diluted 1:500 (Invitrogen, Karlsruhe, Germany) for $1 \mathrm{~h}$. Cells were washed four times with PBS, allowed to settle on polyL-lysine (Sigma-Aldrich, Hamburg, Germany)-coated cover slips and fixed with $4 \%$ paraformaldehyde in PBS for $30 \mathrm{~min}$. The cover slips were washed, dried and mounted with Fluoprep (bioMerieux, France). Slides were examined, and images were acquired in a fluorescence microscope (Axiovert $200 \mathrm{M}$; Carl Zeiss) using AxioVision Version 4.7 software.

Two-dimensional immunoelectrophoresis

Two-dimensional immunoelectrophoresis was performed as previously described [18].

Flow cytometric analyses of $\mathrm{C} 3$ on $\mathrm{RBC}$

DVs and RBC were incubated at a 2:1 ratio in autologous plasma for $1 \mathrm{~h}$ at $37^{\circ} \mathrm{C}$. Cells were washed free of DVs and blocked with $3 \%$ BSA in phosphate-buffered saline (PBS) for $30 \mathrm{~min}$ at room temperature (RT). To $2 \times 10^{7} \mathrm{RBCs}$ in $1 \mathrm{ml}$ of PBS were added $20 \mu \mathrm{l}$ polyclonal rabbit anti-human C3d (DakoCytomation Gmbh, Hamburg, Germany). After $1 \mathrm{~h}$ at RT, cells were washed four times with PBS and incubated with Alexa Fluor 488-conjugated goat anti-rabbit IgG diluted 1:250 (Invitrogen, Karlsruhe, Germany) for $1 \mathrm{~h}$ at RT. RBCs were washed three times with PBS, and flow cytometric analyses were performed using a FACScan flow cytometer (BD Biosciences).

\section{Western blot analyses}

After incubation of RBCs with or without DVs, the reaction mixtures were layered on top of 30,45 and $60 \%$ Percoll step gradients (GE-Healthcare Munich, Germany) solution and centrifuged at $2,500 \times g$ for $15 \mathrm{~min}$ at $4{ }^{\circ} \mathrm{C}$ in a swinging bucket rotor SW $40 \mathrm{Ti}$ (Beckman Coulter, Optima ${ }^{\mathrm{TM}} \mathrm{LE}-80 \mathrm{~K}$ ultracentrifuge). RBCs were recovered from the bottom of the tubes and washed twice with VBS, and RBC ghosts were prepared by lysing the cells with $5 \mathrm{mM}$ phosphate buffer pH.8.0 and $1 \mathrm{mM}$ EDTA [20] in the presence of protease inhibitors. RBC ghosts were solubilized with SDS loading buffer, reduced with $\beta$-mercaptoethanol and boiled at $95^{\circ} \mathrm{C}$ for $10 \mathrm{~min}$. Proteins were separated by SDS-PAGE in $10 \%$ gels. To detect iC $3 \mathrm{~b}$, Western blots were developed by probing with a 1:2,000 dilution of primary polyclonal rabbit antihuman C3d antibody, followed by 1:2,000 diluted secondary HRP-conjugated goat anti-rabbit IgG antibody (Santa Cruz biotechnology). To detect DV proteins, blots were probed with IgG isolated from a pool of high-titered sera containing antibodies against $P$. falciparum [18] (diluted 1:500) and developed with secondary HRP-conjugated goat anti-human IgG. Bands were visualized by chemiluminescence [14].

Hemophagocytosis assays

Opsonized RBCs were offered to macrophages at a ratio of $5 \mathrm{RBC}: 1$ macrophage. The duration of the phagocytosis assays vary according to the target RBCs. As known from the literature [21], coating of RBC solely with $\mathrm{C} 3 \mathrm{~b}$ promotes protracted uptake by macrophages. In pilot experiments, this was found to require $8-10 \mathrm{~h}$ under our conditions. Once phagocytosed, RBCs remain intact for $3 \mathrm{~h}$ and are then digested [22]. Therefore, phagocytosis assays with non-parasitized RBCs were conducted overnight. In contrast, parasitized RBCs are quite rapidly phagocytosed via CD36-dependent and CD36-independent mechanisms. When parasitized cells were employed, it was empirically found that the additional effect of complement opsonization could best be discerned after $60 \mathrm{~min}$. Following the incubation with macrophages, non-phagocytosed RBCs were removed with lysis buffer $\left(150 \mathrm{mM} \mathrm{NH}_{4} \mathrm{Cl}, 7.5 \mathrm{mM}\right.$ $\mathrm{KHCO}_{3}, \mathrm{pH}$ 7.2) for $7 \mathrm{~min}$. After one further wash, cover slips were transferred to microscope slides, Giemsastained, and hemophagocytosis was quantified by viewing 350-500 macrophages and expressed as the percentage of cells that had ingested at least one RBC.

Detection of reactive oxygen species (ROS) generation

Twenty microliters of $50 \%$ RBC were loaded with $0.4 \mathrm{mM}$ $2^{\prime}, 7^{\prime}$ dichlorofluorescin diacetate (DCFH-DA) (SigmaAldrich, Hamburg, Germany) for $30 \mathrm{~min}$ at $37{ }^{\circ} \mathrm{C}$ and then washed four times with VBS. The cells were incubated in $200 \mu$ lautologous plasma with DVs at a ratio of 2DV: $1 \mathrm{RBC}$ at $37^{\circ} \mathrm{C}$ as described above. After $30 \mathrm{~min}$, DVs were removed by washing at $400 \times g$ for $40 \mathrm{~s}$ three times. As a positive control, DCFH-DA-laden RBCs were exposed to $1 \mathrm{mM} \mathrm{H}_{2} \mathrm{O}_{2}$ [23]. Cells were analyzed by flow cytometry. 
Analysis of 4-HNE conjugates by FACS

4-HNE conjugates on the surface of RBC were detected by flow cytometry using rabbit polyclonal antibodies against 4-HNE [11] (Abcam plc, 330 Cambridge Science park, UK). $1 \times 10^{7}$ washed RBCs were incubated with rabbit anti-4-HNE (diluted 1:200) in $0.1 \mathrm{ml} \mathrm{VBS}$ for $1 \mathrm{~h}$ at room temperature, followed by 1-h incubation with Alexa594-conjugated goat anti-rabbit IgG (diluted 1:500). As a positive control, late-stage parasitized RBCs were enriched to $>80 \%$ and stained as above. Cells were analyzed by flow cytometry.

Immunohistochemistry, polarization microscopy and imaging

Brain paraffin sections from autopsies performed at the Department of Pathology, Mahidol University Bangkok, on seven patients who had died of cerebral malaria were immunohistochemically stained for C3d and C5b-9 with the use of the C5b-9 neoantigen-specific monoclonal antibody clone $979 / 142$ produced in this laboratory and a polyclonal antibody against human C3d (DakoCytomation) as described [24]. The same sections were investigated by polarization microscopy, and the images were stacked by CombineZM (http://hadleyweb.pwp.blueyonder.co.uk).

\section{Results}

Deposition of activated C3 on bystander erythrocytes

Incubation of RBCs in autologous plasma caused no C3 conversion and no cellular C3 deposition (Fig. 1a). Presence of DVs in the samples provoked C3 conversion (Fig. 1b). We have reported that the activating particles become coated with $\mathrm{C} 3$ and C5b-9 [14, 18]. Now, the striking finding was made that focal deposits of $\mathrm{C} 3$ also became detectable on the bystander RBCs (Fig. 1b). C3 staining did not derive from adhering DV debris: Western blots developed with pooled, high-titered antisera containing antibodies that recognized DV proteins failed to reveal the presence of parasite proteins on the RBC membranes (not shown).

Upon generation, nascent $\mathrm{C} 3 \mathrm{~b}$ and $\mathrm{C} 5 \mathrm{~b}-9$ must rapidly bind to their targets, failing which irreversible inactivation occurs [25]. The focal distribution of $\mathrm{C} 3$ on bystander cells suggested that, due to its half-life of microseconds and binding range of just $30 \mathrm{~nm}$ [26], attachment of the activated component could occur only at sites that directly contacted the convertase-bearing particles. Two-compartment experiments were therefore conducted. When DVs and RBC were separated by a filter, C3 conversion occurred in the DV compartment and cleavage products diffused through the filter to reach the lower, RBC-containing compartment. However, C3 could not be detected on these cells (Fig. 1c).

Since bystander effects should be magnified on paroxysmal nocturnal hemoglobinuria (PNH)-RBCs lacking CD55 and CD59 [27], experiments were undertaken using RBCs from 4 patients with PNH. Incubation in autologous plasma with DVs caused substantive lysis of these cells. Notably, this could not have been provoked by simple adherence of complement-bearing DV debris. No hemolysis was observed in the two-compartment experiments (Fig. 2a). By flow cytometry, no C3d staining was detectable on cells that had been incubated in plasma alone, or on RBCs that had been separated from the DVs by a filter (Fig. 2b). However, following coincubation with DVs, all cells stained positively, heterogeneity possibly stemming from varying numbers of DV "hits." When cells from a patient with PNH were analyzed, a population of intensely stained cells possibly representing PNH-RBCs was detected (Fig. 2b).

Western blot analyses corroborated these findings. DVmediated C3 conversion led to appearance of the $63 \mathrm{kDa}$ iC $3 \mathrm{~b} \alpha$ band [28] in plasma (Fig. 2c, compare lanes 2 and 3 ). The activation product was absent on control PNHRBCs incubated in plasma alone (Fig. 2c, lane 4) but present on membranes of cells that had been coincubated with DVs (Fig. 2c, lane 5).

Bystander deposition of $\mathrm{C} 3$ promotes erythrophagocytosis

It is known that deposition of $\mathrm{C} 3 \mathrm{~b}$ alone on $\mathrm{RBCs}$ promotes their removal from the circulation [29], but that macrophage uptake of such cells is protracted [21, 30]. We surmised that this might explain why C3-bearing RBCs can escape splenic elimination and are thus detectable in peripheral blood of malarial patients [12, 13]. Phagocytosis experiments were next performed. Uptake of C3-bearing cells by neutrophil granulocytes or macrophages could not be detected within $4 \mathrm{~h}$. However, interesting findings emerged when the phagocytosis assays were prolonged. While all controls remained negative even after overnight culture (Fig. 3a, left panel), 5-8 \% erythrophagocytosis was observed with normal opsonized RBCs (Fig. 3a, right panel), and respective rates rose to 15-20\% with $\mathrm{PNH}-$ RBCs (Fig. 3b). Erythrophagocytosis was complement dependent and never occurred following incubation of RBCs and DVs in heat-inactivated plasma.

No evidence that DVs promote erythrophagocytosis via alternative mechanisms

Experiments were performed to exclude that DVs alter RBCs by alternative mechanisms. Free hemozoin can provoke peroxidation of polyunsaturated membrane fatty acids 
A
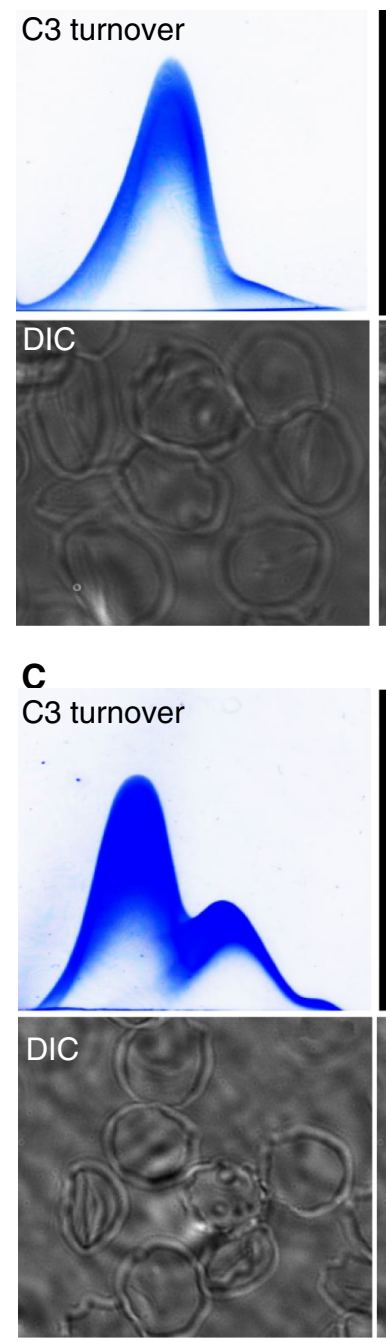
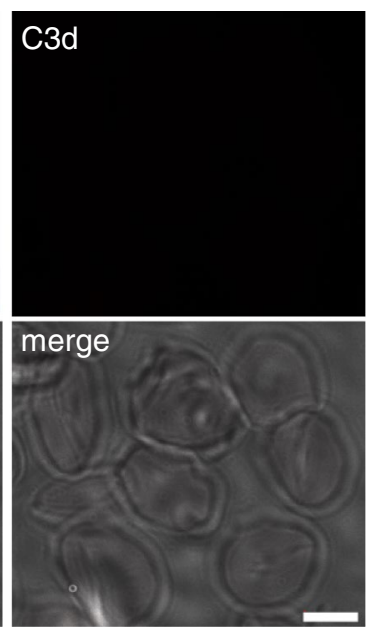

C3d
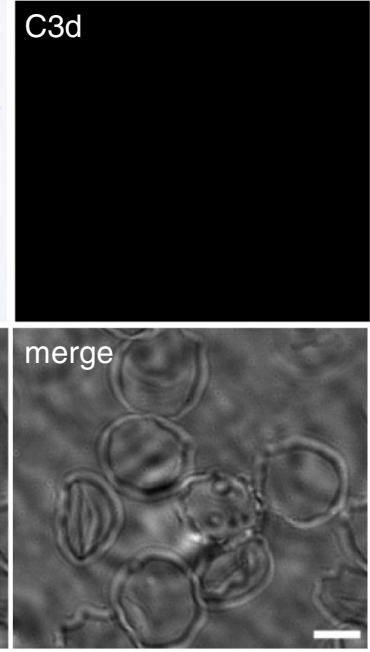

Fig. 1 DVs mediate contact-dependent complement deposition on bystander cells. a RBCs were incubated in autologous plasma in the absence of DVs. Two-dimensional immunoelectrophoresis showed absence of $\mathrm{C} 3$ turnover (first dimension electrophoresis: left to right; second dimension: bottom to top). Cells were stained for bound $\mathrm{C} 3$ and viewed by differential interference contrast (DIC) or fluorescence microscopy. C3 was undetectable on the cells. b Coincubation of RBCs with DVs resulted in C3 turnover in the plasma (arrow).

with generation of aldehyde-4-hydroxynenal (HNE), which can be transferred to neighboring cells and promote their phagocytosis [11]. When RBCs were incubated with DVs, however, production of reactive oxygen species (ROS) could not be discerned (Fig. 4a) and no HNE adducts were detectable (Fig. 4b, c). DV ingestion has been reported to cause macrophage activation [31]. Uptake in these cells is complement independent [31] and occurred in our control samples wherein coincubation of RBCs with DVs was undertaken in heat-inactivated plasma. Hemozoin uptake reportedly induced TNF-alpha in human macrophages [31], but this was not observed with our DV preparations

\section{B}
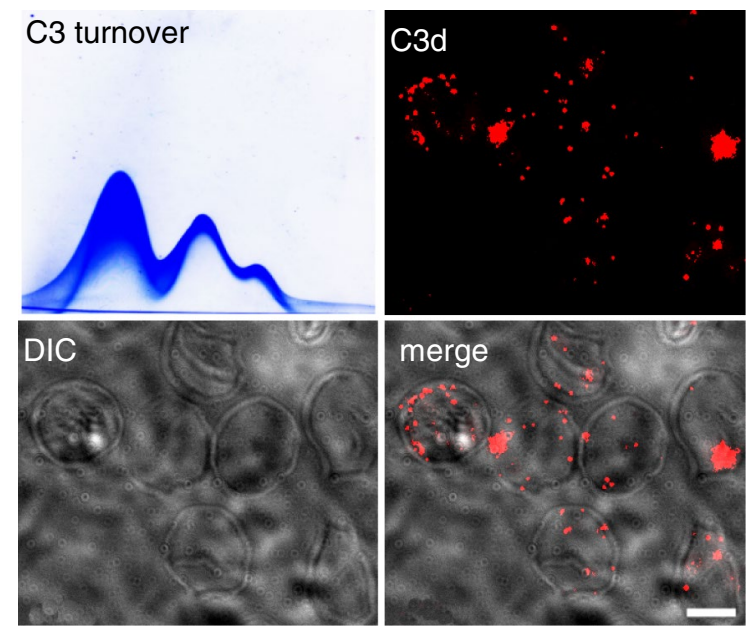

Merged phase contrast and fluorescence images revealed focal deposits of $\mathrm{C} 3$ on the cells. $\mathbf{c}$ RBCs in the lower compartment of a culture well were separated by a filter from DVs in the upper compartment. Diffusion of converted C3 to the RBC compartment was ascertained by immunoelectrophoresis of a sample from the lower compartment. However, no C3 deposits were detectable on the cells. Scale bars $5 \mu \mathrm{m}$

(unpublished data), whose uptake provoked no erythrophagocytosis without complement. Erythrophagocytosis also was not observed when DVs and RBCs were separated in the two-compartment experiments (data not shown).

Enhanced bystander complement deposition on parasitized cells

During parasite invasion and development, GPI-anchored RBC proteins including CD59 traffic to the parasitophorous vacuole membrane [32]. To determine whether this might magnify bystander complement attack, cultures 


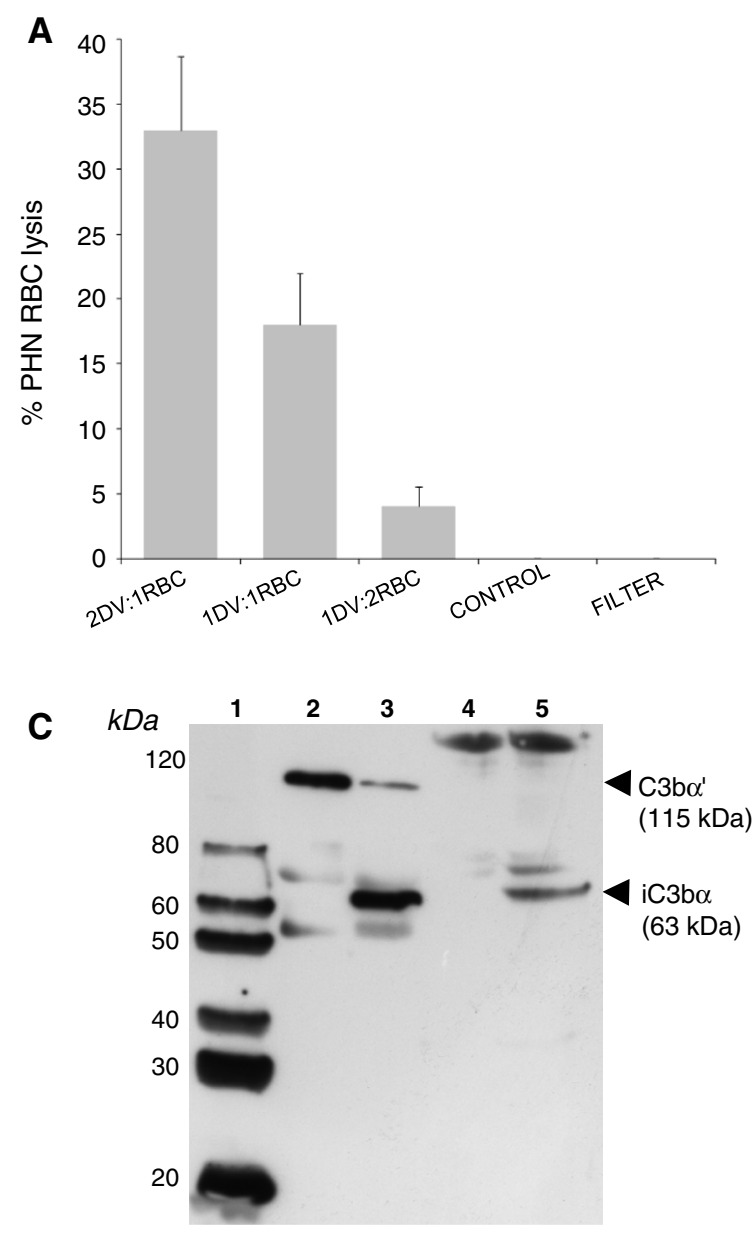

Fig. 2 Identification of bystander-deposited activated complement components. a DVs and RBCs from patients with PNH were coincubated at the given ratios, and hemolysis was determined after $60 \mathrm{~min}$, $37{ }^{\circ} \mathrm{C}(n=4, \pm \mathrm{SD})$. Control cells were incubated without DVs. The filter experiment was performed as in Fig. 1c, and hemolysis was read in the lower RBC compartment. b Flow cytometric analysis of RBCs from a healthy donor stained for bound $\mathrm{C} 3$ following incubation in plasma (green), plasma and DVs (black), or plasma and filter-separated DVs (blue). Similar analyses of RBCs from a patient with $\mathrm{PNH}$ following incubation in plasma (yellow) or plasma and DVs (red). c

were prepared using RBCs of the same donors above, and ring-stage infected cells were employed as targets. CD36mediated macrophage uptake of these cells occurs spontaneously [10], oxidized lipids providing additional signals for erythrophagocytosis [11]. Complement effects were here revealed by shortening experiments to $60 \mathrm{~min}$. Less than $20 \%$ erythrophagocytosis was noted with control cells that had been incubated either in active plasma without DVs (Fig. 5, left panel), or in heat-inactivated plasma with DVs. However, DV-mediated bystander opsonization led to erythrophagocytosis rates approaching $100 \%$ (Fig. 5, right panel). The majority of phagocytosed cells were reduced in size, resembling what has recently been reported as pitting of phagocytosed schizonts [33]. These findings showed

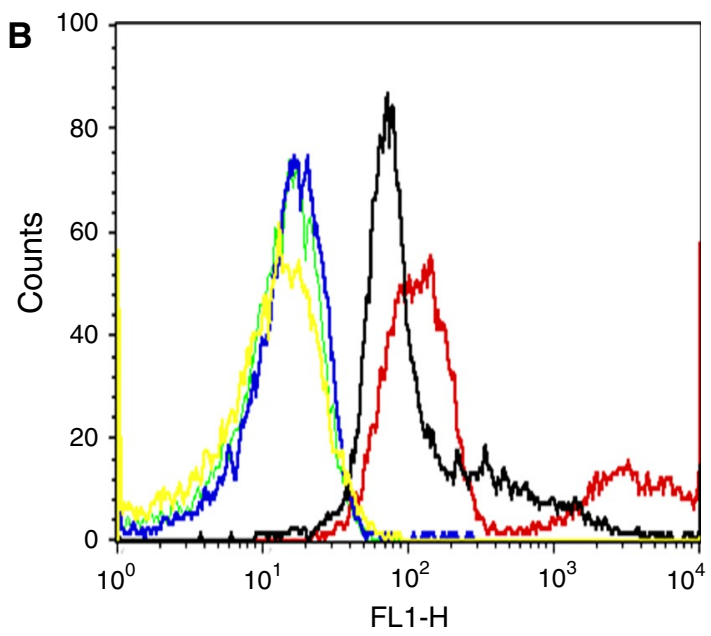

Plasma samples and PNH-RBC membranes obtained from the experiment of Fig. 2a were subjected to Western blot analyses. Lane 1 molecular weight marker. Lane 2 control plasma sample incubated with autologous RBCs without DVs showing presence of uncleaved $\mathrm{C} 3 \alpha$. Lane 3 similar sample additionally spiked with DVs showing appearance of iC $3 \mathrm{~b} \alpha 63 \mathrm{kDa}$ fragment in the plasma. Lane 4 membranes from RBC incubated without DVs showing absence of iC $3 b \alpha$ $63 \mathrm{kDa}$. Lane 5 membranes of RBCs following coincubation with DVs, showing presence of $\mathrm{iC} 3 \mathrm{~b} \alpha 63 \mathrm{kDa}$ (color figure online)

that several independent factors could synergize to promote erythrophagocytosis of infected cells.

Colocalization of DVs and activated complement components with sequestered erythrocytes in brain capillaries

In order to obtain an indication whether the described scenarios might occur in vivo, brain paraffin sections from six autopsies of patients who had died of cerebral malaria were analyzed by immunohistochemistry for the presence of activated C3 and C5b-9 and, in parallel, by polarized light microscopy for detection of hemozoin. Indeed, C3d and the assembled terminal C5b-9 complex could be detected 

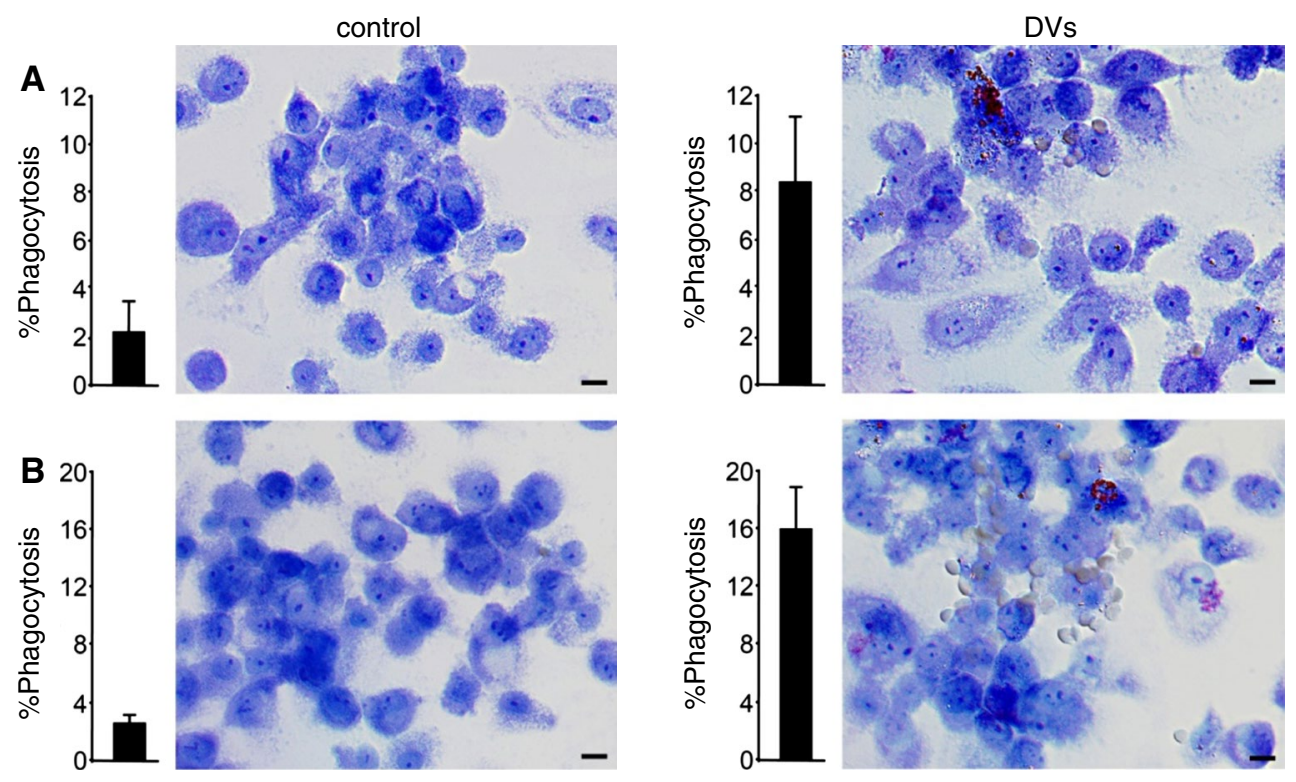

Fig. 3 Bystander complement deposition provokes erythrophagocytosis by human macrophages. a, b Left panels examples for controls wherein RBCs were incubated in autologous plasma alone; right pan-

els examples for experiments wherein RBCs were coincubated with DVs. a Normal RBCs. b PNH-RBCs $(n=4 \pm \mathrm{SD})$
Fig. 4 Absence of ROS production and HNE adducts provoked by contact of DVs with RBCs. a Cells were loaded with DCFHDA, and fluorescence was noted upon exposure to $\mathrm{H} 2 \mathrm{O} 2$. In contrast, no fluorescence was observed upon incubation of cells with DVs (blue curve superimposable on control, red). b Immunocytological staining of HNE adducts showing staining of positive controls (green) compared with negative cells (red). c No staining for HNE was noted on RBCs following incubation with DVs in active or inactive plasma (color figure online)
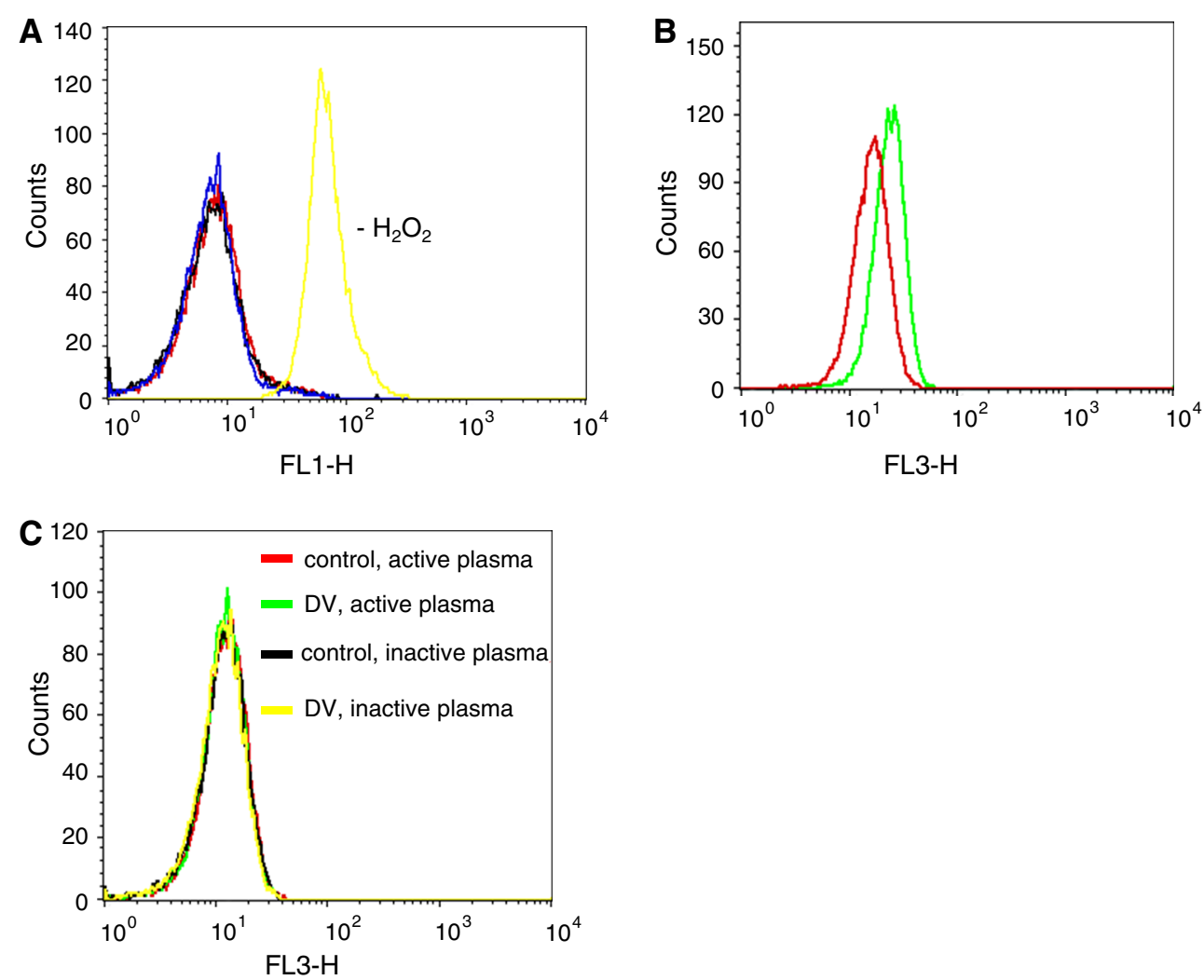

in all sections and importantly, they colocalized with and often extended locally beyond the massive accumulations of DVs, which were readily identifiable under polarized light (Fig. 6). The monoclonal antibody against C5b-9 recognizes a neoantigen that is only present on the assembled terminal complex, and positive immunohistochemical staining thus reveals that complement activation has taken place and occurred to completion at the given sites. 
Fig. 5 Enhanced erythrophagocytosis of opsonized, ring-stage infected RBCs. Left panel unopsonized control and right panel opsonized RBCs. Percent phagocytosis denotes the percentage of phagocytosing macrophages in the respective experiments $(n=4 \pm \mathrm{SD})$
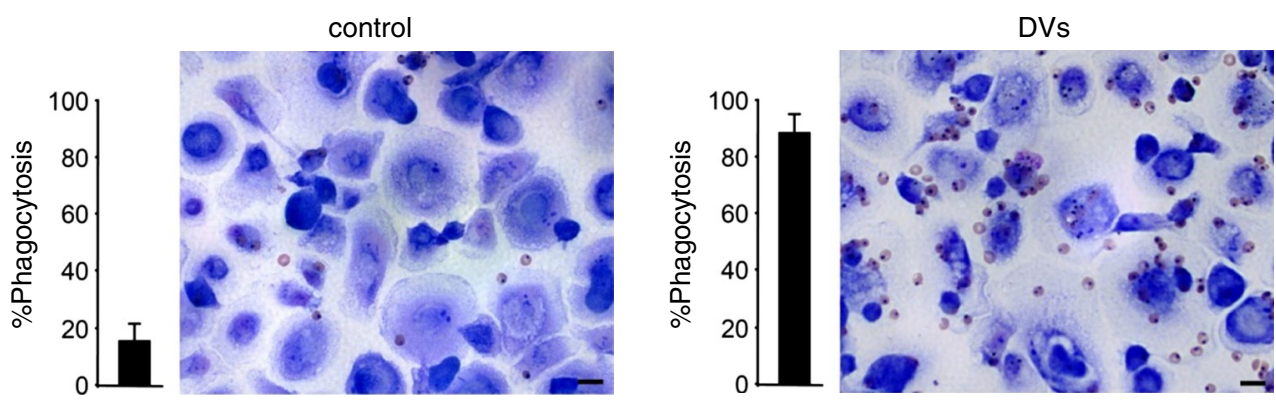
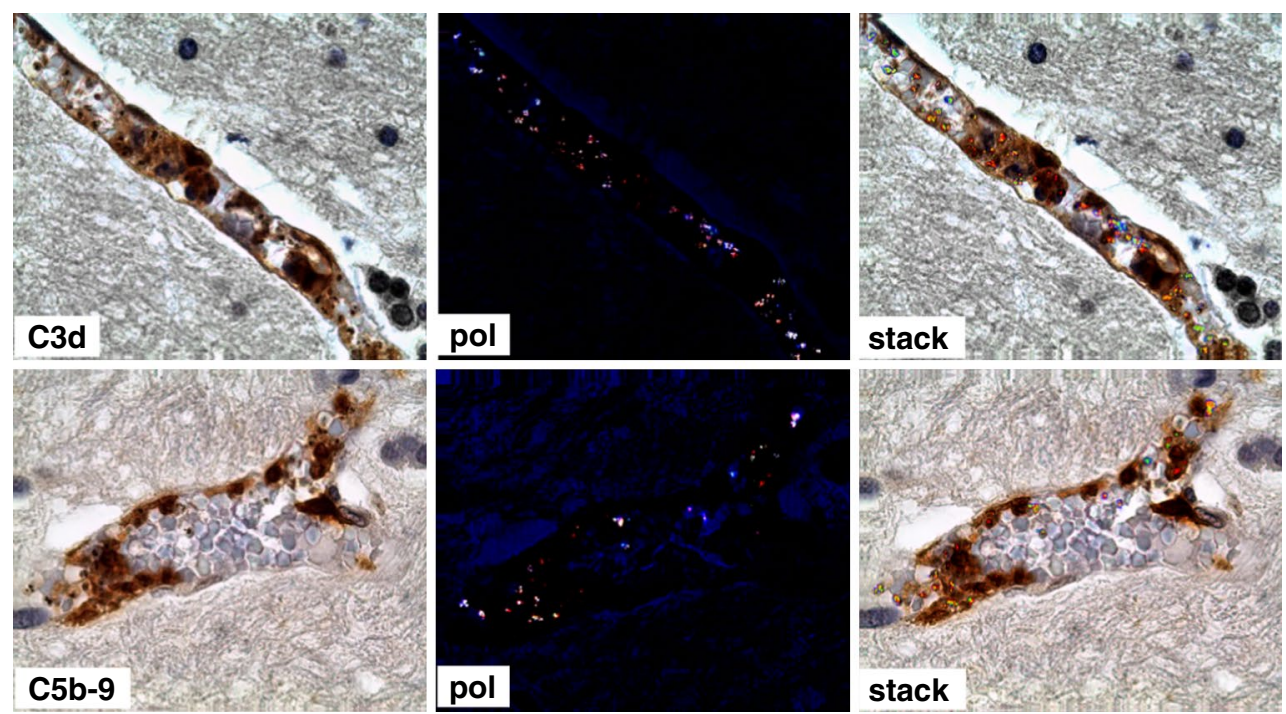

Fig. 6 Immunohistochemical staining of $\mathrm{C} 3 \mathrm{~d}$ and $\mathrm{C} 5 \mathrm{~b}-9$ on brain sections of a patient with severe cerebral malaria. Note strong C3d (upper panels) and C5b-9 (lower panels; brown) staining in cortical capillaries (left panels) and both extra- and intracellular malaria pigment (hemozoin) distinguished by polarized light microscopy in the same sections (middle panels). The right panels (stacking of the right

\section{Discussion}

The results of this investigation lead to a new concept regarding the pathogenesis of anemia in falciparum malaria. Due to their propensity to potently activate the alternative complement pathway, DVs that are released into the circulation during the rupture of parasitized erythrocytes become heavily coated with $\mathrm{C} 3$-convertases. When these particles collide with host cells, the latter become subject to bystander attack and activated complement components are then deposited on their surface. This will promote $\mathrm{C} 3$-dependent macrophage phagocytosis. While the model experiments conducted herein were performed with erythrocytes, the results may naturally also extend to platelets. A simple concept emerges to explain why life spans of uninfected blood cells are shortened in patients with severe falciparum malaria.

$\mathrm{C} 3$ and $\mathrm{C} 5$ convertases assembled on alternative pathway activator surfaces generate $\mathrm{C} 3 \mathrm{~b}$ and $\mathrm{C} 5 \mathrm{~b}$ molecules whose and middle panels by CombineZM, see "Materials and methods") further illustrate the close intermingling and colocalization of complement activation products and malaria pigment (hemozoin) on the one hand and extension of the staining of both complement products over the area occupied by malaria pigment (hemozoin) on the other hand. Magnification $\times 630$ (color figure online)

short half-lives generally restrict their action to the activating particle itself $[25,26]$. Two requirements theoretically need to be met in order for bystander "attack" on innocent cells to occur: (1) high density of the convertases leading to vigorous generation of the activated components (2) close contact between the activator surface and bystander, which will enable a fraction of nascent $\mathrm{C} 3 \mathrm{~b}$ and $\mathrm{C} 5 \mathrm{~b}-9$ to escape fluid-phase inactivation and bind to the target. The fate of the bystander cell will subsequently depend on the presence or absence of complement regulatory molecules and inhibitors. CD55 alias decay-accelerating factor (DAF) promotes dissociation ("decay") of the convertases, while CD59 inhibits insertion of C5b-9 complexes into the lipid bilayer $[25,27]$. Both represent key complement inhibitors of the erythrocyte. Their absence on PNH cells accounts for the hemolytic episodes which occur when complement is spontaneously activated in patients' plasma. Delineation of these events showed that bystander attack by complement 
occurring from the fluid phase is held in check through the presence of the GPI-anchored inhibitors [27].

That bystander attack might analogously be mounted from a solid phase was reported by our group decades ago [34]. Erythrocytes and neutrophil leukocytes were shown to represent susceptible targets in blood passing through oxygenators that at that time were equipped with complementactivating membranes. Since then, the concept of bystander complement attack from solid phase activators has occasionally resurfaced in the literature, but no in-depth investigation has been made with any defined particulate activator. In this regard, the present report fills a long-standing conceptual gap.

The evidence for contact transfer of activated complement components mediated by the DV is compelling. Upon coincubation of DVs with RBCs in autologous plasma, punctuate staining of $\mathrm{C} 3$ on the cells became apparent, which indicated the presence of clusters of $\mathrm{C} 3$ on the surfaces. The size of the clusters was compatible with the idea that they were generated at sites of collision with DVs. Filter experiments then showed that $\mathrm{C} 3$ deposition required such contact: When DVs and RBCs were physically separated, no binding of $\mathrm{C} 3$ occurred although complement activation took place to the same extent in the compartment containing the DVs. This finding accorded with the long-known fact that activated C3 has a very short half-life, which is why binding to innocent bystander targets usually does not occur [25].

That C3 bound to the bystander cells in its activated state was apparent from Western blot analyses, which identified the $63 \mathrm{kDaC} 3$ product that is generated through the cleavage and activation of the native molecule [26]. Again, binding was completely prevented by separating the cells from the activating particles.

Experiments utilizing PNH-RBCs corroborated and extended these results. These cells lack both CD55 (DAF) and CD59 (C5b-9 inhibitor) and would therefore be expected to be particularly vulnerable to bystander complement attack [27]. This was the case: Not only was C3 deposition enhanced, a fraction of the cells even underwent C5b-9-mediated lysis. The latter was also contact dependent, as was expected because of the known short half-life of nascent C5b-9 complexes [35]. This finding provided conclusive evidence that activated complement components had bound directly to the RBCs, because membraneinserted C5b-9 cannot be transferred to bystander cells, and simple absorption of complement-coated DVs or DV debris could not have caused hemolysis.

Activated C3 is generally considered to represent a major opsonin. Erythrophagocytosis experiments were therefore conducted, and the results merit special comment. RBCs were incubated with DVs at a ratio of just 1:1 in autologous plasma. After removal of the bulk of
DVs, the cells were seeded onto macrophages and erythrophagocytosis rates were determined. The first experiments, which were conducted over the conventional period of 1-4 h, yielded entirely negative results, and no effects of bystander $\mathrm{C} 3$-opsonization could be discerned. We then became aware of earlier investigations that had shown macrophage uptake of particles opsonized solely with $\mathrm{C} 3 \mathrm{~b}$ to be inefficient and protracted [21, 30]. Thereupon, phagocytosis experiments were prolonged overnight, and positive results were obtained. Erythrophagocytosis rates in controls remained in the order of $2 \%$ but rose to $6-10 \%$ with opsonized cells. These rates doubled when PNH-RBC were used as targets.

At this juncture, some very interesting reports in the literature appeared under a new light. In elegant studies, Lauer et al. [32] demonstrated that during parasite invasion and development, GPI-anchored RBC proteins including CD59 traffic from the cell surface to the parasitophorous vacuole membrane. Reduction of CD59 and, by extrapolation, of CD55 at the RBC surface would be expected to confer a PNH-like phenotype onto the cells. To test this contention, erythrophagocytosis experiments were performed with ring-stage infected RBCs. The results were impressive. It is known that CD36-mediated macrophage uptake of these cells occurs spontaneously [10] and that oxidized membrane lipids further augment this process [11]. The additional effect of bystander C3 deposition was revealed through shortening of experiments to $1 \mathrm{~h}$. Erythrophagocytosis rates were below $20 \%$ in controls, but approached $100 \%$ with opsonized cells.

Direct evidence in support of the proposed concept emerged through immunohistochemical analyses of brain sections from autopsies of patients who had died of severe malaria. Sections were stained for activated C3 and C5b-9 and analyzed by polarized light microscopy in parallel. In the first investigation of this kind, myriads of hemozoin crystals were detected in the brain capillaries, in striking colocalization with activated complement. C5b-9 staining was undertaken with a monoclonal antibody that specifically recognizes the assembled C5b-9 complex. The findings thus provide irrefutable evidence that complement activation takes place at sites of DV liberation. Thereby, complement deposition appeared often to extend to neighboring RBCs, possibly reflecting contact-dependent bystander attack. Such stainings were never observed on RBCs that were located distant to the DVs.

Recent published data from animal experiments are in line with the concept that has emerged from the present investigation. Life spans of RBCs from non-infected mice were shortened when transfused into infected animals [36]. Vice versa, however, non-parasitized RBCs from infected 


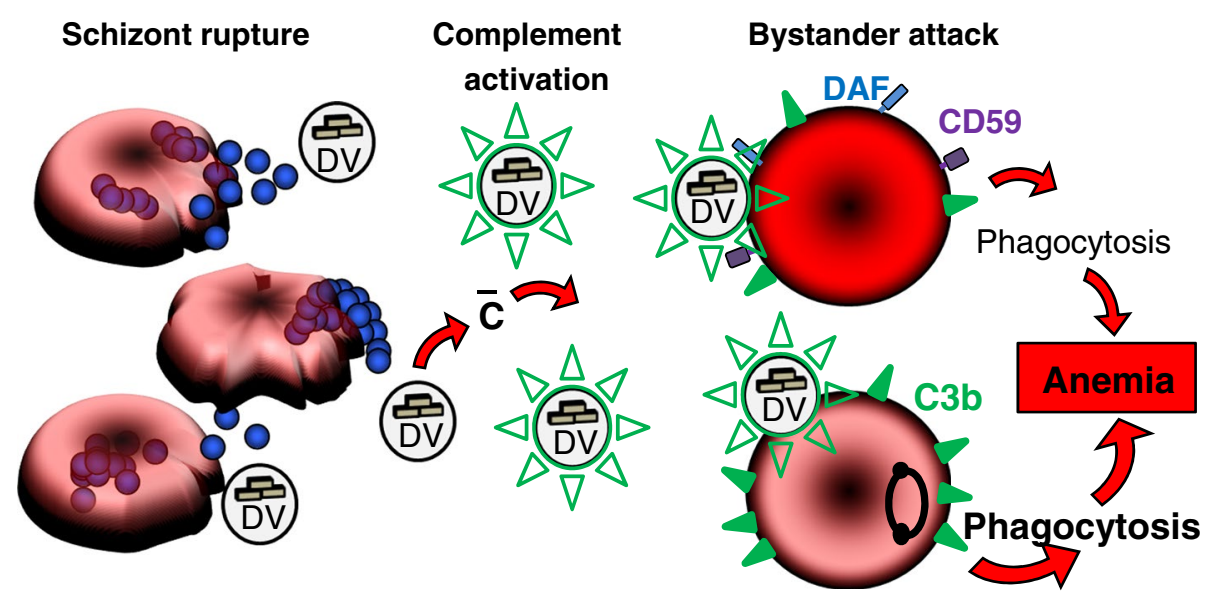

Fig. 7 Proposed principle of contact-dependent bystander complement attack. Schizont rupture liberates one DV alongside with infected merozoites. Alternative pathway C3/C5 convertase assembles on the DV, which then continuously generates C3b and C5b-9. A fraction of the active components bind to the DV, but the bulk undergoes rapid inactivation in the fluid phase. Because of the short life span of the molecules, bystander binding of nascent C $3 b$ and C5b-9 is only possible when the DV collides with the target cell. Complement inhibitors, in particular CD55 and CD59, counter the extent and efficacy of bystander attack. Ring-stage infected RBCs are phagocytosed even more effectively due to their reduced CD55 and CD59 expression animals had normal life spans in healthy recipients. These findings are expected since the majority of opsonized cells would be continuously removed in the infected animals and not be available for transfusion. The opposite observation indicates that, as argued here, the shortened life spans of uninfected RBCs are antibody independent and incurred by agents generated and present in the infected host.

Figure 7 schematically illustrates the proposed principle of contact-dependent complement opsonization that marks cells for antibody-independent phagocytosis. Note that the numeric ratios of $\mathrm{DV}: \mathrm{RBC}$ at any given location are possibly not the critical factor: rather, the number of total "hits" an erythrocyte acquires during its passage through the microcirculation likely decides its fate. Elements that counteract bystander complement attack including factor $\mathrm{H}$ and $\mathrm{RBC}$-bound regulators will thereby come into play. In $\mathrm{PNH}$ patients, hypersusceptibility to DV-mediated bystander attack may predispose to anemia on the one hand, but conversely subserve a protective function by promoting premature lysis of infected cells. Clearly, many dynamic variables are likely to influence the duration and extent of bystander complement attack, and the contribution of this process to development of malarial anemia in the individual patient.

Acknowledgments The beginning of this work was supported by the Deutsche Forschungsgemeinschaft, Sonderforschungsbereich 490 (S.B.), CRC877 (K.R.), the Cluster of Excellence "Inflammation at Interfaces" (K.R.) and the Thai Infectious Disease Network (S.C.B, S.B.). The review panel of the Deutsche Forschungsgemeinschaft then dedicated time and effort to evaluate the ongoing project, which was assigned a low priority and therefore terminated. This work includes parts of the doctoral thesis of S.D.H.

\section{References}

1. Ekvall H (2003) Malaria and anemia. Curr Opin Hematol 10(2):108-114

2. Lamikanra AA, Brown D, Potocnik A, Casals-Pascual C, Langhorne J, Roberts DJ (2007) Malarial anemia: of mice and men. Blood 110(1):18-28

3. Buffet PA, Safeukui I, Deplaine G, Brousse V, Prendki V, Thellier M, Turner GD, Mercereau-Puijalon O (2011) The pathogenesis of Plasmodium falciparum malaria in humans: insights from splenic physiology. Blood 117(2):381-392

4. Skudowitz RB, Katz J, Lurie A, Levin J, Metz J (1973) Mechanisms of thrombocytopenia in malignant tertian malaria. Br Med J 2(5865):515-518

5. Looareesuwan S, Davis TM, Pukrittayakamee S, Supanaranond W, Desakorn V, Silamut K, Krishna S, Boonamrung S, White NJ (1991) Erythrocyte survival in severe falciparum malaria. Acta Trop 48(4):263-270

6. Jakeman GN, Saul A, Hogarth WL, Collins WE (1999) Anaemia of acute malaria infections in non-immune patients primarily results from destruction of uninfected erythrocytes. Parasitology 119(Pt 2):127-133

7. Pongponratn E, Riganti M, Bunnag D, Harinasuta T (1987) Spleen in falciparum malaria: ultrastructural study. Southeast Asian J Trop Med Public Health 18(4):491-501

8. Park TS, Oh SH, Choi JC, Kim HH, Chang CL, Son HC, Lee EY (2003) Plasmodium vivax malaria complicated by hemophagocytic syndrome in an immunocompetent serviceman. Am J Hematol 74(2):127-130

9. Ohnishi K, Mitsui K, Komiya N, Iwasaki N, Akashi A, Hamabe Y (2007) CLINICAL case report: falciparum malaria with hemophagocytic syndrome. Am J Trop Med Hyg 76(6):1016-1018

10. Ayi K, Patel SN, Serghides L, Smith TG, Kain KC (2005) Nonopsonic phagocytosis of erythrocytes infected with ring-stage Plasmodium falciparum. Infect Immun 73(4):2559-2563

11. Uyoga S, Skorokhod OA, Opiyo M, Orori EN, Williams TN, Arese P, Schwarzer E (2012) Transfer of 4-hydroxynonenal from parasitized to non-parasitized erythrocytes in rosettes. Proposed role in severe malaria anemia. Br J Haematol 157(1):116-124 
12. Greenwood BM, Stratton D, Williamson WA, Mohammed I (1978) A study of the role of immunological factors in the pathogenesis of the anaemia of acute malaria. Trans R Soc Trop Med Hyg 72(4):378-385

13. Helegbe GK, Goka BQ, Kurtzhals JA, Addae MM, Ollaga E, Tetteh JK, Dodoo D, Ofori MF, Obeng-Adjei G, Hirayama K, Awandare GA, Akanmori BD (2007) Complement activation in Ghanaian children with severe Plasmodium falciparum malaria. Malar J 6:165

14. Dasari P, Heber SD, Beisele M, Torzewski M, Reifenberg K, Orning C, Fries A, Zapf AL, Baumeister S, Lingelbach K, Udomsangpetch R, Bhakdi SC, Reiss K, Bhakdi S (2012) Digestive vacuole of Plasmodium falciparum released during erythrocyte rupture dually activates complement and coagulation. Blood 119(18):4301-4310

15. Klouche M, Gottschling S, Gerl V, Hell W, Husmann M, Dorweiler B, Messner M, Bhakdi S (1998) Atherogenic properties of enzymatically degraded LDL: selective induction of MCP-1 and cytotoxic effects on human macrophages. Arterioscler Thromb Vasc Biol 18(9):1376-1385

16. Trager W, Jensen JB (1976) Human malaria parasites in continuous culture. Science 193(4254):673-675

17. Lambros C, Vanderberg JP (1979) Synchronization of Plasmodium falciparum erythrocytic stages in culture. J Parasitol 65(3):418-420

18. Dasari P, Reiss K, Lingelbach K, Baumeister S, Lucius R, Udomsangpetch R, Bhakdi SC, Bhakdi S (2011) Digestive vacuoles of Plasmodium falciparum are selectively phagocytosed by and impair killing function of polymorphonuclear leukocytes. Blood 118(18):4946-4956

19. Kutner S, Breuer WV, Ginsburg H, Aley SB, Cabantchik ZI (1985) Characterization of permeation pathways in the plasma membrane of human erythrocytes infected with early stages of Plasmodium falciparum: association with parasite development. J Cell Physiol 125(3):521-527

20. Schwoch G, Passow H (1973) Preparation and properties of human erythrocyte ghosts. Mol Cell Biochem 2(2):197-218

21. Aderem A, Underhill DM (1999) Mechanisms of phagocytosis in macrophages. Annu Rev Immunol 17:593-623

22. Loegering DJ, Commins LM, Minnear FL, Gary LA, Hill LA (1987) Effect of Kupffer cell phagocytosis of erythrocytes and erythrocyte ghosts on susceptibility to endotoxemia and bacteremia. Infect Immun 55(9):2074-2080

23. Amer J, Goldfarb A, Fibach E (2003) Flow cytometric measurement of reactive oxygen species production by normal and thalassaemic red blood cells. Eur J Haematol 70(2):84-90
24. Bhakdi S, Torzewski M, Paprotka K, Schmitt S, Barsoom H, Suriyaphol P, Han SR, Lackner KJ, Husmann M (2004) Possible protective role for $\mathrm{C}$-reactive protein in atherogenesis: complement activation by modified lipoproteins halts before detrimental terminal sequence. Circulation 109(15):1870-1876

25. Muller-Eberhard HJ (1988) Molecular organization and function of the complement system. Annu Rev Biochem 57:321-347

26. Sim RB, Twose TM, Paterson DS, Sim E (1981) The covalentbinding reaction of complement component C3. Biochem J 193(1):115-127

27. Parker CJ (1996) Molecular basis of paroxysmal nocturnal hemoglobinuria. Stem Cells 14(4):396-411

28. Stover CM, Luckett JC, Echtenacher B, Dupont A, Figgitt SE, Brown J, Mannel DN, Schwaeble WJ (2008) Properdin plays a protective role in polymicrobial septic peritonitis. J Immunol 180(5):3313-3318

29. Karnchanaphanurach P, Mirchev R, Ghiran I, Asara JM, Papahadjopoulos-Sternberg B, Nicholson-Weller A, Golan DE (2009) $\mathrm{C} 3 \mathrm{~b}$ deposition on human erythrocytes induces the formation of a membrane skeleton-linked protein complex. J Clin Invest 119(4):788-801

30. Mollison PL (1986) Survival curves of incompatible red cells. An analytical review. Transfusion 26(1):43-50

31. Schwarzer E, Turrini F, Ulliers D, Giribaldi G, Ginsburg H, Arese $P$ (1992) Impairment of macrophage functions after ingestion of Plasmodium falciparum-infected erythrocytes or isolated malarial pigment. J Exp Med 176(4):1033-1041

32. Lauer S, VanWye J, Harrison T, McManus H, Samuel BU, Hiller NL, Mohandas N, Haldar K (2000) Vacuolar uptake of host components, and a role for cholesterol and sphingomyelin in malarial infection. EMBO J 19(14):3556-3564

33. Anyona SB, Schrier SL, Gichuki CW, Waitumbi JN (2006) Pitting of malaria parasites and spherocyte formation. Malar J 5:64

34. Salama A, Hugo F, Heinrich D, Hoege R, Mueller R, Kiefel V, Mueller-Eckhart C, Bhakdi S (1988) Deposition of terminal C5b-9 complement complexes on erythrocytes and leukocytes during cardiopulmonary bypass. N Engl J Med 318(7):408-414

35. Bhakdi S, Hugo F, Tranum-Jensen J (1990) Functions and relevance of the terminal complement sequence. Blut 60(6):309-318

36. Evans KJ, Hansen DS, van Rooijen N, Buckingham LA, Schofield L (2006) Severe malarial anemia of low parasite burden in rodent models results from accelerated clearance of uninfected erythrocytes. Blood 107(3):1192-1199 